\title{
A New Method for the Detection of Singular Points in Fingerprint Images
}

\author{
Filipe Magalhães \\ INESC Porto, Portugal \\ filipe.magalhaeseinescporto.pt
}

\author{
Hélder P. Oliveira \\ INESC Porto, Portugal \\ helder.oliveira@inescporto.pt
}

\author{
Aurélio C. Campilho \\ INEB, Porto, Portugal \\ campilhoefe.up.pt
}

\begin{abstract}
Automatic biometric identification based on fingerprints is still one of the most reliable identification method in criminal and forensic applications. A critical step in fingerprint analysis without human intervention is to automatically and reliably extract singular points from the input fingerprint images. These singular points (cores and deltas) not only represent the characteristics of local ridge patterns but also determine the topological structure (i.e., fingerprint type) and largely influence the orientation field. Poincaré Indexbased methods are one of the most common for singular points detection. However, these methods usually result in many spurious detections. Therefore, we propose an enhanced version of the method presented by Zhou et al. [13] that introduced a feature called DORIC to improve the detection. Our principal contribution lies in the adoption of a smoothed orientation field and in the formulation of a new algorithm to analyze the DORIC feature. Experimental results show that the proposed algorithm is accurate and robust, giving better results than the best reported results so far, with improvements in the range of 5\% to $7 \%$.
\end{abstract}

\section{Introduction}

Fingerprint images are directionally oriented patterns formed by ridges and valleys that can be captured from a finger with a multitude of sensors, particularly, capacitive, optical, thermal sweeping and ultrasonic. Fingerprints are unique to each individual and have long been widely used as a biometric identification tool [10]. The singular points cores and deltas - are the most important topological global characteristics of a fingerprint [12]. The singular point area is defined as a region where the ridge curvature is higher than normal and where the direction of the ridge changes rapidly.

These singular points can constitute a powerful basis for the classification of fingerprint types [5], as well as for fingerprint alignment and orientation field modeling [4, 11].

Several methods for detecting and analyzing singular points in fingerprint images have been devised $[1,7]$.
However, the most widely used tool for singularity extraction is the Poincaré Index, since it is relatively simple to compute and robust to image rotation. The first application of Poincaré indices to fingerprint images was presented by Kawagoe and Tojo [7].

Poincaré Index-based methods consider the discontinuous orientation distribution around singular points, by computing the sum of the orientation changes along a closed circle around a point to judge whether it is a singular point. However, many spurious detections usually arise, deriving from low quality images, scars, smudges, etc. that exhibit orientation patterns closely resembling a true singular point. Even after postprocessing, many of these spurious detections still remain since only local information is used. Therefore, to accurately distinguish the genuine singular points, global discriminative information should be used in the detection process.

In order to address these problems we adopt the following strategy: 1) to calculate the Poincaré Index using a smoothed orientation field, in order to reduce the number of spurious points detected; 2) remove some spurious points, regarding some core-delta topological constraints, with the use of postprocessing steps; and 3) to use a recent feature called DORIC (Differences of the ORIentation values along a Circle) [13], which provides more discriminative information that can be further used to remove remaining spurious points.

Experimental results showed that the proposed algorithm is accurate and robust for a wide variety of fingerprint types. Compared with previous research, we achieved better detection results with this new method.

The rest of this paper is organized as follows: Section 2 provides an insight into the mathematical background of the Poincaré Index and describes in detail the several parts of the proposed algorithm. In Section 3 the principal results are presented as well as a comparison between the state-of-the-art and the proposed algorithm. These results are analyzed and discussed in Section 4 and, finally, some conclusions are presented. 


\section{Methods}

In this section the methods used in the devised strategy for the detection of singular points in fingerprint images will be presented.

\subsection{Poincare Index mathematical background}

A singularity can be defined as a local region of the fingerprint where the ridge pattern has special properties making it visually prominent. To identify the principal singular points on a fingerprint, i. e. cores and deltas, a Poincaré Index based method can be used.

In Figure 1 it is presented the vector field and the Poincaré Index of three typical patterns.
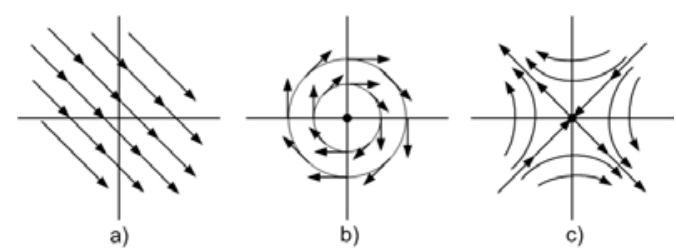

Figure 1: Three typical vector field patterns. a) No singularity, $I(\gamma)=0$. b) Circle (Core), $I(\gamma)=1$. c) Saddle (Delta), $I(\gamma)=-1$.

A core is defined by a turning point of an inner-most ridge and a delta is a place where two ridges running sideby-side diverge. Using the Poincaré Index, a value of +1 can be assigned to a core, a value of -1 to a delta and 0 to a non-singularity.

Let us consider $V(x, y)=\Phi_{x}(x, y)+i \cdot \Phi_{y}(x, y)$, where $V(x, y)$ is a continuous $2 \mathrm{D}$ vector field, $\Phi_{x}(x, y)$ and $\Phi_{y}(x, y)$ are its real and imaginary components, respectively. The Poincaré Index $I(\gamma)$ of $V(x, y)$ along closed path $\gamma$ can be computed by:

$$
I(\gamma)=\frac{1}{2 \cdot \pi} \int_{(x, y) \in \gamma} d \phi(x, y),
$$

where $\phi(x, y)$ represents the angle on each $(x, y)$ point of the vector field along $\gamma$ and $\phi(x, y) \in[0,2 \pi[$. Usually, the integration is taken counterclockwise.

\subsection{Proposed algorithm}

In Figure 2 it can be seen the algorithm's block diagram. In the following subsections its principal steps are described.

\subsubsection{Normalization}

In this step the input image is normalized in order to have a specific mean and standard deviation. The main purpose of this step is the contrast enhancement to reduce the variations of the gray-level values along ridges and valleys, to

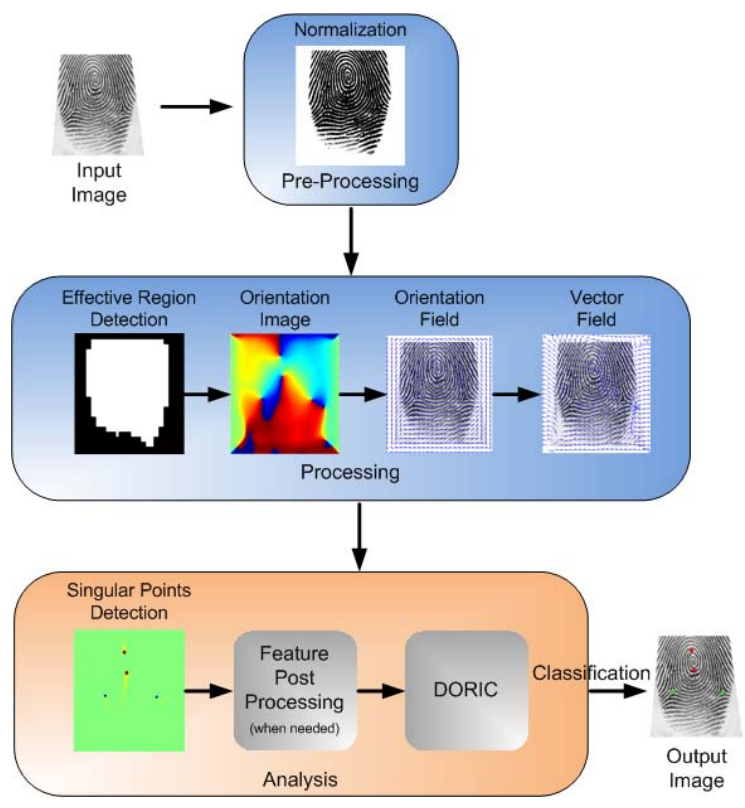

Figure 2: Proposed algorithm flowchart.

facilitate the next processing steps, particularly the orientation field computation.

Let $I$ be a gray-level image and $I(x, y)$ the pixel value at the $(x, y)$ position. $\mu$ denotes the estimated mean and $\sigma$ the estimated standard deviation of $I$. The normalization procedure is applied to the image pixels using the following equation [2]:

$I_{n}(x, y)= \begin{cases}\mu_{n}+\sqrt{\frac{\sigma_{n}(I(x, y)-\mu)^{2}}{\sigma}}, & \text { if } I(x, y)>\mu \\ \mu_{n}-\sqrt{\frac{\sigma_{n}(I(x, y)-\mu)^{2}}{\sigma}}, & \text { otherwise, }\end{cases}$

where $I_{n}(x, y)$ represents the value for each normalized gray-level pixel, and $\mu_{n}$ and $\sigma_{n}$ the desired normalized mean and standard deviation values, respectively.

\subsubsection{Effective region detection}

In order to reduce the size of the region to be processed and, consequently, the computational time, an effective region detection algorithm was implemented. Within this algorithm, as usual in image processing, the normalized image was broken into blocks of size $\left(B_{z} \times B_{z}\right)$, and the mean and the standard deviation of each block were evaluated. If the standard deviation was above a threshold $t_{\sigma}$ it was deemed part of the fingerprint.

In this process some morphological operators were also included, such as, erosion and flood-fill. The erosion was applied in first place in order to create a "safety zone", preventing the posterior occurrence of spurious detections on the borders of the fingerprints. 


\subsubsection{Smoothed orientation field}

The orientation image represents an intrinsic property of the fingerprint images and defines invariant orientation regions for ridges and valleys in a local neighborhood. Many methods were devised for the computation of the orientation field and many are supported by the fact that fingerprints can be seen as an oriented texture [6,7]. In this work, we chose to compute the orientation field using a least mean square orientation estimation [3] (see equation (3)).

$$
\begin{array}{r}
\theta(x, y)=\frac{1}{2} \cdot \tan ^{-1}\left(\frac{\sum_{x=1}^{W} \sum_{y=1}^{W} \Psi_{1}(x, y)}{\sum_{x=1}^{W} \sum_{y=1}^{W} \Psi_{2}(x, y)}\right) \\
\Psi_{1}(x, y)=2 \cdot G_{x}(x, y) \cdot G_{y}(x, y) \\
\Psi_{2}(x, y)=G_{x}^{2}(x, y)-G_{y}^{2}(x, y),
\end{array}
$$

where $\theta(x, y)$ is the least square estimate of the local ridge orientation at the block with size $W \times W$, centered at pixel $(x, y) . G_{x}(x, y)$ and $G_{y}(x, y)$ represent the gradient along the $x$ and $y$ directions, respectively.

Due to the presence of noise, corrupted ridge and valley structures in the input image, the estimated local ridge orientation, $\theta(x, y)$, may not always be correct. Since local ridge orientation varies slowly in a local neighborhood where no singular points appear, a low-pass filter can be used to modify the incorrect local ridge orientation. In order to smooth the initial orientation field a Gaussian filter was implemented with size $G_{z}$ and standard deviation equal to $\sigma_{g}[2,13]$.

After the computation of the orientation field for each pixel, the vector field $V(x, y)$ can be computed using the following equation:

$$
V(x, y)=\cos (2 \cdot \theta(x, y))+i \cdot \sin (2 \cdot \theta(x, y)),
$$

In Figure 3 it can be seen some results for the computation of the orientation image and respective non-smoothed and smoothed orientation fields and vector field.

\subsubsection{Singularity detection using the Poincaré Index}

The pixels can be defined as cores, deltas or nonsingularities depending on their Poincaré Index values.

The Poincaré Index method can usually detect nearly all true singular points, when it is computed in a small region, but this can also lead to the detection of too many spurious points. If the detection is made into a very large region, true singular points could be missed. To increase the correct detection rate and reduce the detection of spurious points it

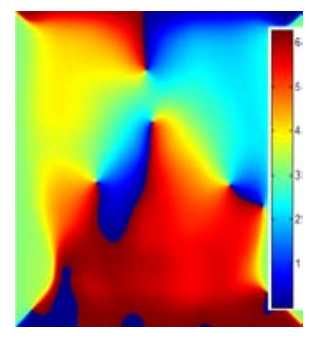

(a) Orientation image $[0,2 \pi[$.

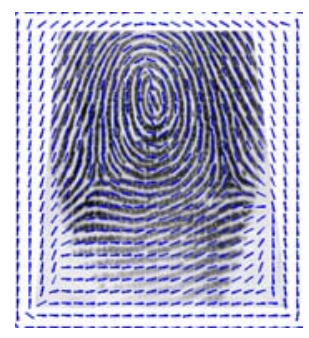

(c) Smoothed orientation field.

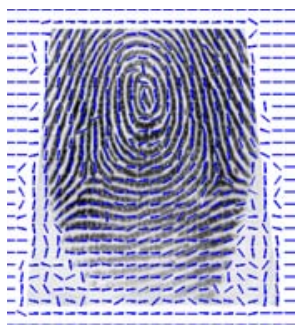

(b) Non-smoothed orientation field.

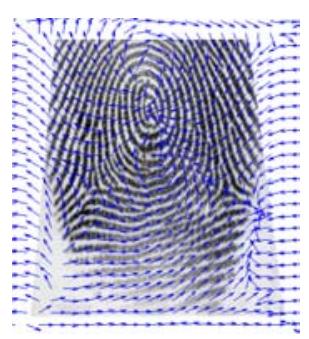

(d) Vector field.
Figure 3: Orientation image (a) and respective orientation fields (non-smoothed (b) and smoothed (c)) and vector field (d).

is necessary to establish a compromise in the choice of the optimal region size.

For the computation of the Poincaré Index value $\left(I_{P}\right)$, a circle, with a specific radius $\left(r_{p}\right)$ and $N_{p}$ points, is centered in each pixel of the fingerprint effective region. Then, the Poincaré Index is computed as the sum of the differences between the orientations of two consecutive points in the circle $\left(o_{i+1}\right.$ and $\left.o_{i}\right)$, as stated in the following equation, being that a counterclockwise direction is assumed:

$$
I_{P}=\frac{1}{\pi} \sum_{i=1}^{N-1} f\left(o_{i+1}-o_{i}\right)=\frac{1}{\pi} \sum_{i=1}^{N-1} f\left(\delta o_{i}\right),
$$

where $o_{N}=o_{1}, o_{i} \in[0, \pi[$, and $f$ is defined as:

$$
f(x)= \begin{cases}-x, & |x| \leq \frac{\pi}{2} \\ \pi-x, & x>\frac{\pi}{2} \\ \pi+x, & x<-\frac{\pi}{2}\end{cases}
$$

Figure 4 presents the output image after the Poincaré Index method step.

\subsubsection{Feature postprocessing}

After the singular points detection using the Poincaré Index, as previously stated, some spurious points may still remain. In order to reduce the amount of possibly false detected points a postprocessing step was introduced. 


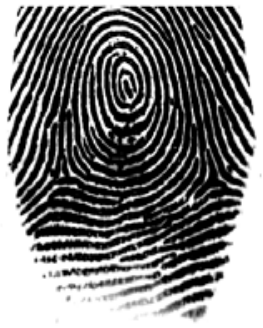

(a) Normalized image with fingerprint.

Figure 4: Detection of the singular points in image (a) using the Poincaré Index. The cores are represented in red while the deltas are represented in blue.

In the first place, if two or more singular points of the same type, cores or deltas, are close to each other, within a certain distance $(d)$, they become to be represented by a centroid. The second postprocessing step, determines if a core and delta are too close to each other. If they are closer than a certain distance $\left(d_{s}\right)$ both points are removed.

During the experimental tests, we noticed that these steps were rarely used. This is supported by the use of the smoothed orientation field, and, therefore, they will only be needed in very particular occasions, for instance, when in the presence of low-quality images, or fingerprints with scars, which introduce strong "artifacts" to the orientation field. If no smoothing is applied, this postprocessing phase reveals itself to be very important in the removal of spurious points.

\subsubsection{DORIC feature}

In order to remove spurious detections while preserving a good detection rate, Zhou et al. [13] proposed a novel feature extended from the Poincaré Index, which can be used to verify the trueness of each detection after using the Poincaré Index and the postprocessing algorithms.

The Poincare Index is the sum of $\delta o_{i}$ and it does not describe completely a singular point. When there are creases, scars, or smudges in the fingerprint images, the Poincaré Index will result in the detection of spurious singular points that even after the postprocessing are not removed.

Similar to the Poincaré Index method, the DORIC feature is computed along a closed circle, centered in each of the detected singular points, with a specific radius $\left(r_{d}\right)$ and $N_{d}$ points.

The novel feature, called DORIC, consists of all the $\delta o_{i}$, and, therefore, it can describe the singular points more completely. Since the orientation field is defined in $[0, \pi[$, the DORIC feature will exhibit one positive pulse, with height near $\pi$, for a core and a negative pulse, with height near $-\pi$, for a delta. Although the noise around the true singular points may alter the curves a little, it can be clearly distinguished the difference between true and spurious singular points. Two DORIC features of true singular points are illustrated in Figure 5.

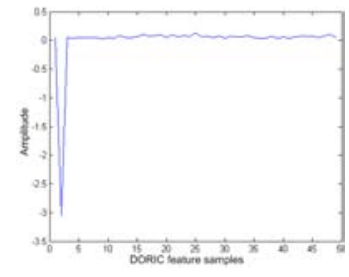

(a) DORIC feature for a true delta.

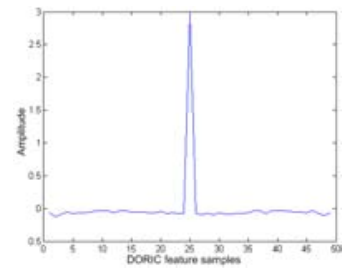

(b) DORIC feature for a true core.
Figure 5: DORIC features of two true singular points.

After the postprocessing phase, there is only one singular point for any fairly large region. Thus, it is more adequate to compute the DORIC features along a large circle. This enables the use of a higher number of points in the circle which will cause the DORIC feature curves to become more continuous and much easier to analyze.

Finally, to classify the remaining singular points as true or spurious points, the following algorithm was devised:

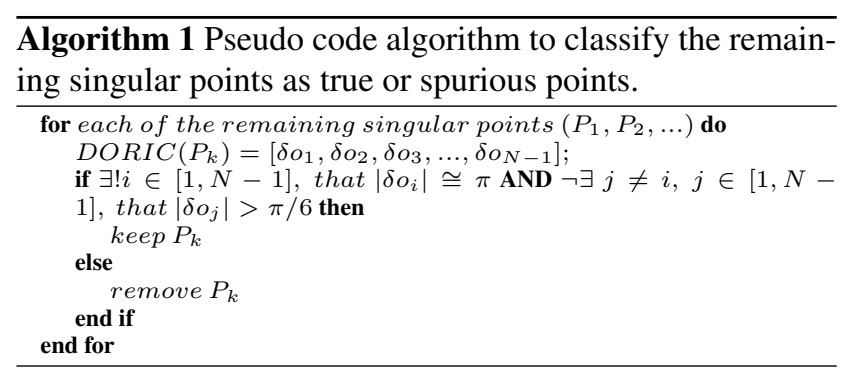

\section{Experimental results}

The proposed method was tested on four public databases (FVC02 DB1 and DB2 [8] and FVC04 DB1 and DB2 [9]) and one database created by the authors. Each FVC database has 80 images, relative to 10 fingers and 8 prints of each finger. The database created by the authors consists of 70 images, corresponding to 1 image of each finger from 7 individuals. These images were acquired with an optical sensor (Microsoft Fingerprint Reader - model 1033) without any special effort on image quality and cleaning procedures.

For the validation of the detection results, the authors independently analyzed each image and registered the occurrence of cores and deltas. If there was some disagreement between their decisions, a joint decision would be made.

For the results presented, the detection rate is defined as the ratio of truly detected singular points to all ground truth singular points. The miss rate is defined as the ratio of the number of missed singular points to the number of all ground truth singular points. The false alarm rate is defined 
as the number of falsely detected singular points versus the number of all ground truth singular points. If all singular points are detected and there are no spurious singular points in a fingerprint, the fingerprint is said to be "correctly" detected [13].

During the implementation phases, all the parameters were experimentally tuned using the FVC02 DB1 as a "training" set. The most adequate values found are presented in TABLE 1.

\begin{tabular}{|c|c|c|c|}
\hline Method & \multicolumn{2}{|c|}{ Parameter } & Value \\
\hline \hline \multirow{2}{*}{ Normalization } & mean & $\mu_{n}$ & 0 \\
\cline { 2 - 4 } & stdev & $\sigma_{n}$ & 1 \\
\hline \multirow{2}{*}{ Effec. region detect. } & block size & $B_{z}$ & 16 \\
\cline { 2 - 4 } & stdev threshold & $t_{\sigma}$ & 0.3 \\
\hline \multirow{3}{*}{ Smoothed orient. field } & block size & $W$ & 10 \\
\cline { 2 - 4 } & Gaussian stdev & $\sigma_{g}$ & 10 \\
\cline { 2 - 4 } & Gauss. block size & $G_{z}$ & 60 \\
\hline \multirow{2}{*}{ Sing.points detection } & radius & $r_{p}$ & 5 \\
\cline { 2 - 4 } & number points & $N_{p}$ & 8 \\
\hline \multirow{3}{*}{ Feat.postprocess. } & dist. core - core & $d$ & 16 \\
\cline { 2 - 4 } & dist. delta - delta & $d$ & 16 \\
\cline { 2 - 4 } & dist. core - delta & $d_{s}$ & 20 \\
\hline \multirow{2}{*}{ DORICfeature } & radius & $r_{d}$ & 10 \\
\cline { 2 - 4 } & number points & $N_{d}$ & 50 \\
\hline
\end{tabular}

Table 1: Values of the parameters used in the algorithm.

When possible, the obtained results were compared to those exhibited by Zhou et al. [13], as they state their work presented better results than the best reported ones.

In Table 2, 3, 4 and 5, the obtained results are presented (all values are in percentage).

\begin{tabular}{|c|c|c|c|}
\hline & & Proposed & Zhou's [13] \\
\hline \hline \multirow{3}{*}{ Cores } & Detection rate & 100 & 95.78 \\
\cline { 2 - 4 } & Miss rate & 0 & 4.22 \\
\cline { 2 - 4 } & False alarm rate & 1.25 & 2.27 \\
\hline \multirow{3}{*}{ Deltas } & Detection rate & 97.50 & 96.98 \\
\cline { 2 - 4 } & Miss rate & 2.50 & 3.02 \\
\cline { 2 - 4 } & False alarm rate & 1.25 & 9.97 \\
\hline \multicolumn{2}{|c|}{ Fingerprint precision } & 95.00 & 88.88 \\
\hline
\end{tabular}

Table 2: Comparison results of different detection algorithms on FVC02's DB1.

\begin{tabular}{|c|c|c|c|}
\hline & & Proposed & Zhou's [13] \\
\hline \hline \multirow{3}{*}{ Cores } & Detection rate & 98.75 & 95.51 \\
\cline { 2 - 4 } & Miss rate & 1.25 & 4.49 \\
\cline { 2 - 4 } & False alarm rate & 7.50 & 8.45 \\
\hline \multirow{3}{*}{ Deltas } & Detection rate & 95.00 & 90.88 \\
\cline { 2 - 4 } & Miss rate & 5.00 & 9.12 \\
\cline { 2 - 4 } & False alarm rate & 1.25 & 12.54 \\
\hline \multicolumn{2}{|c|}{ Fingerprint precision } & 86.25 & 81.25 \\
\hline
\end{tabular}

Table 3: Comparison results of different detection algorithms on FVC02's DB2.

\begin{tabular}{|c|c|c|c|}
\hline & & $D B 1$ & $D B 2$ \\
\hline \hline \multirow{3}{*}{ Cores } & Detection rate & 100 & 90.00 \\
\cline { 2 - 4 } & Miss rate & 0 & 10.00 \\
\cline { 2 - 4 } & False alarm rate & 3.75 & 17.50 \\
\hline \multirow{3}{*}{ Deltas } & Detection rate & 96.25 & 97.50 \\
\cline { 2 - 4 } & Miss rate & 3.75 & 2.50 \\
\cline { 2 - 4 } & False alarm rate & 1.25 & 8.75 \\
\hline \multicolumn{2}{|c|}{ Fingerprint precision } & 92.50 & 67.50 \\
\hline
\end{tabular}

Table 4: Results with the proposed algorithm on FVC04's DB1 and DB2.

\begin{tabular}{|c|c|c|}
\hline \multirow{3}{*}{ Cores } & Detection rate & 95.71 \\
\cline { 2 - 3 } & Miss rate & 4.29 \\
\cline { 2 - 3 } Deltas & False alarm rate & 5.71 \\
\hline \multirow{3}{*}{} & Detection rate & 97.14 \\
\cline { 2 - 3 } & Miss rate & 2.86 \\
\cline { 2 - 3 } & False alarm rate & 2.86 \\
\hline \multicolumn{2}{|c|}{ Fingerprint precision } & 84.29 \\
\hline
\end{tabular}

Table 5: Results with the proposed algorithm on the authors' database.

Some images of the results obtained with the proposed algorithm are presented in Figure 6.

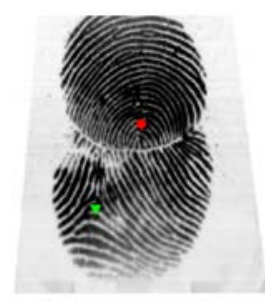

(a)

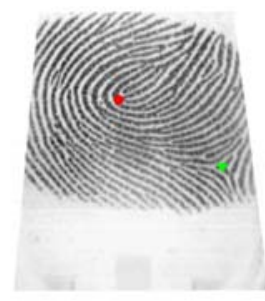

(c)

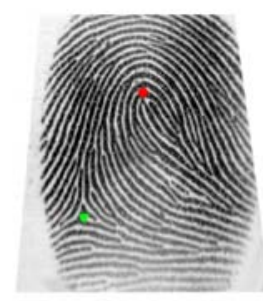

(b)

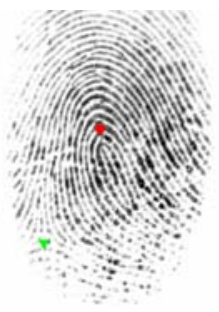

(d)
Figure 6: Results obtained with the proposed algorithm for different types of fingerprint images and topologies.

The results were obtained with $M A T L A B^{\mathrm{R}}$ with an Intel ${ }^{\circledR} C$ ore $^{T M} 2$ Duo CPU $@ 2.50 G H z$ and $3 G B$ of $R A M$. In average the processing time was approximately 10 seconds for each fingerprint image with sizes ranging from $296 \times 560$ to $640 \times 480$, depending on the database.

\section{Analysis and discussion}

From the analysis of Figure 6, it can be seen that our algorithm behaves well for a wide variety of images and fingerprint types, even in the presence of scars (Figure 6(a)), rotations (Figure 6(b) and 6(c)) and low-quality images (Figure 6(d)).

However, there are some cases where the algorithm fails. For instance, in Figure 7(a) it is missing one delta. This can be justified by the fact that the Poincaré Index calculation did not have revealed any singularity where expected (see Figure 7(b).

In Figure 8(a), we can see one spurious delta that was not removed by the proposed algorithm. Its DORIC feature can be seen in Figure 8(b) and it can be seen that it closely resembles the trace of a true delta, therefore, it was not possible for the proposed algorithm to remove it. 


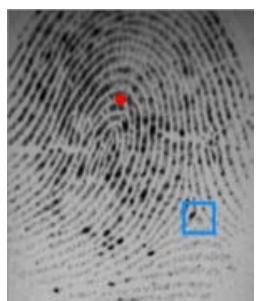

(a) Detected singular points marked on the fingerprint image.

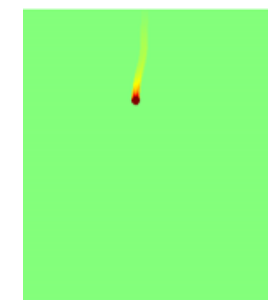

(b) Poincaré Index image.
Figure 7: Missed detection of a singular point (Delta) by the Poincare Index. The blue square marks the expected location of the missed delta.

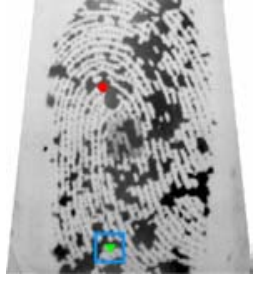

(a) Detected singular points marked on the fingerprint image.

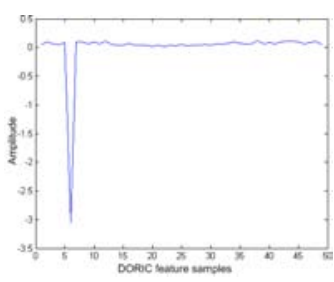

(b) DORIC feature of the spurious delta.
Figure 8: One example of a spurious delta that was not removed with the use of the DORIC feature. The blue square identifies the over detected delta.

From the results of Table 2 and 3 it can be stated that, relatively to the $\mathrm{FVC0} 2$ databases, the proposed algorithm promoted the achievement of better results than those presented by Zhou et al. [13]. Relatively to the results obtained for the remaining databases (see Table 4 and 5), it can be said that the results were also very good.

\section{Conclusion}

This work presented a new method for the detection of singular points in fingerprint images. The proposed algorithm has two fundamental parts: 1) detection of singularities using the Poincare Index on a smoothed orientation field, which contributed since the beginning to the reduction of the number of spurious points detected; and 2) use the DORIC feature to improve the discriminative ability of the Poincaré Index-based detection.

From the analysis of the obtained results we can state that the main causes of errors are deltas and cores located in the fingerprints' borders, as well as low-quality images, scars, creases, smudges, etc. that will lead to the obtention of erroneous orientation fields, which are crucial to the detection of the singular points.

As a future improvement, some of the parameters, particularly those referring to distances, could be adaptively set. One possible approach would be to detect the width of ridges and valleys and, therefore, proportionally set the parameters. Despite this has not been done yet, the proposed algorithm was able to provide very good results for a wide variety of fingerprint types and images, when compared with other approaches recently published.

\section{Acknowledgements}

The authors would like to thank Fundação para a Ciência e Tecnologia $(F C T)$ for the financial support of the $\mathrm{PhD}$ grants SFRH/BD/45380/2008 and SFRH/BD/43772/2008.

\section{References}

[1] G. A. Drets and H. G. Liljenström. Intelligent biometric techniques in fingerprint and face recognition. CRC Press, New York, 1999.

[2] L. Hong, Y. Wan, and J. A. Fingerprint image enhancement: algorithm and performance evaluation. IEEE Transactions on, Pattern Analysis and Machine Intelligence, 20(8):777789, 1998.

[3] A. Jain, L. Hong, and R. Bolle. On-line fingerprint verification. IEEE Transactions on Pattern Analysis and Machine Intelligence, 19(4):302-314, 1997.

[4] G. Jinwei and Z. Jie. A novel model for orientation field of fingerprints. In Proceedings IEEE Computer Society Conference on Computer Vision and Pattern Recognition, volume 2, pages II-493-8, 2003.

[5] K. Karu and A. K. Jain. Fingerprint classification. Pattern Recognition, 29(3):389-404, 1996.

[6] M. Kass and A. Witkin. Analyzing oriented patterns. Comput. Vision Graph. Image Process., 37(3):362-385, 1987. 27996.

[7] M. Kawagoe and A. Tojo. Fingerprint pattern classification. Pattern Recognition, 17(3):295-303, 1984.

[8] D. Maio, D. Maltoni, R. Cappelli, J. L. Wayman, and A. K. Jain. Fvc2002: Second fingerprint verification competition. In Proceedings 16th International Conference on Pattern Recognition, volume 3, pages 811-814, 2002.

[9] D. Maio, D. Maltoni, R. Cappelli, J. L. Wayman, and A. K. Jain. Fvc2004: Third fingerprint verification competition. In Proceedings International Conference on Biometric Authentication (ICBA), pages 1-7, Hong Kong, 2004.

[10] S. Pankanti, S. Prabhakar, and A. K. Jain. On the individuality of fingerprints. IEEE Transactions on Pattern Analysis and Machine Intelligence, 24(8):1010-1025, 2002.

[11] B. G. Sherlock and D. M. Monro. A model for interpreting fingerprint topology. Pattern Recognition, 26(7):1047-1055, 1993.

[12] V. S. Srinivasan and N. N. Murthy. Detection of singular points in fingerprint images. Pattern Recognition, 25(2):139-153, 1992.

[13] J. Zhou, F. Chen, and J. Gu. A novel algorithm for detecting singular points from fingerprint images. IEEE Transactions on Pattern Analysis and Machine Intelligence, 31(7):12391250, 2009. 